This item was submitted to Loughborough's Research Repository by the author.

Items in Figshare are protected by copyright, with all rights reserved, unless otherwise indicated.

\title{
From faceted classification to knowledge discovery of semi-structured text
} records

PLEASE CITE THE PUBLISHED VERSION

http://www.springer.com/engineering/mathematical/book/978-3-642-01090-3

PUBLISHER

(C) Springer

VERSION

AM (Accepted Manuscript)

LICENCE

CC BY-NC-ND 4.0

REPOSITORY RECORD

Goh, Yee M., Matt Giess, Chris McMahon, and Ying Liu. 2019. "From Faceted Classification to Knowledge Discovery of Semi-structured Text Records". figshare. https://hdl.handle.net/2134/8009. 
This item was submitted to Loughborough's Institutional Repository (https://dspace.lboro.ac.uk/) by the author and is made available under the following Creative Commons Licence conditions.

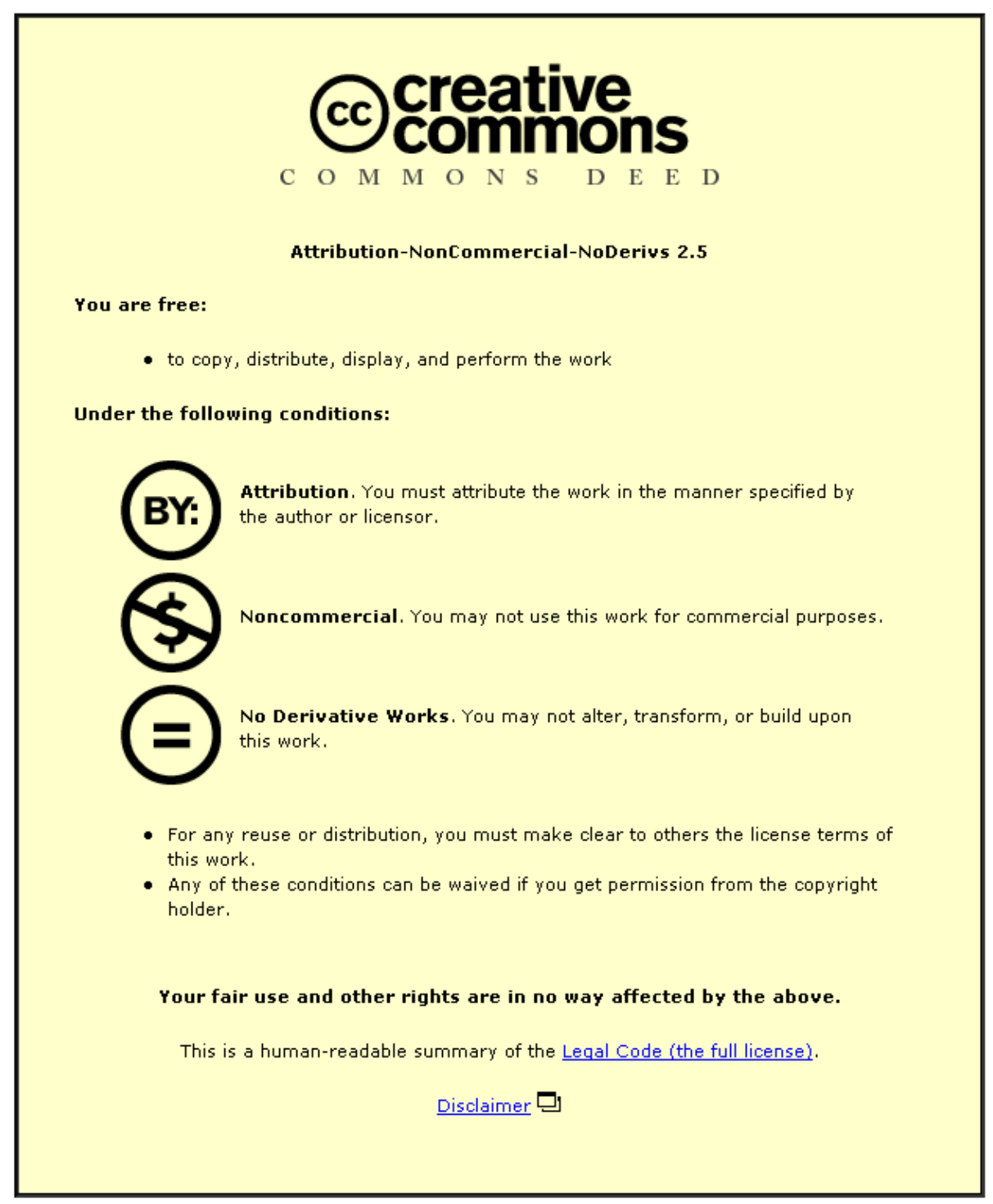

For the full text of this licence, please go to: http://creativecommons.org/licenses/by-nc-nd/2.5/ 


\title{
Faceted Classification to KnOWledge Discovery FROM Semi- STRUCTURED TEXT RECORDS
}

\author{
Yee Mey Goh, Matt Giess, Chris McMahon and Ying Liu
}

\begin{abstract}
The maintenance and service records collected and maintained by the aerospace companies are a useful resource to the in-service engineers in providing their ongoing support of their aircrafts. Such records are typically semi-structured and contain useful information such as a description of the issue and references to correspondences and documentation generated during its resolution. The information in the database is frequently retrieved to aid resolution of newly reported issues. At present, engineers may rely on a keyword search in conjunction with a number of contextual clues to retrieve relevant records from the database. It is believed that further values can be realised from the collection of these records for indicating recurrent and systemic issues which may not have been apparent previously. A faceted classification approach was implemented to enhance the retrieval and knowledge discovery from extensive aerospace in-service records. The retrieval mechanism afforded by faceted classification can expedite responses to urgent in-service issues as well as enable knowledge discovery that could potentially lead to root-cause findings and continuous improvement. The approach can be described as a structured text mining involving records preparation, construction of the classification schemes and data mining.
\end{abstract}

\section{Introduction}

Observation from in-service events can often indicate how well the design of an engineering artefact satisfies the performance, operational and maintenance requirements. Such knowledge is useful to the engineering company in providing maintenance support, repair and upgrade of their existing products as well as in improving the design of their future products. Therefore, information collected from inservice is a useful resource to the engineering companies, particularly aerospace because of the high value and extended life involved. The use of in-service information for decision-making is most evident in condition-based and predictive maintenance using sensors-based data logging and monitoring systems. These systems allow the detection, isolation, identification and prediction of fault condition(s) based on data processing and analysis. Statistical, artificial intelligence, model-based and rule-based approaches are typically employed to algorithmically relate the patterns in data to the expected outcomes in-service (Jardine et al., 2006). As an example, the Distributed Aircraft Maintenance Environment (DAME) is an e-Science project demonstrating the use of GRID infrastructure to implement online health monitoring and fault diagnostic systems for the maintenance of aircraft engines in distributed environments (Ong, 2004).

Besides supporting the ongoing maintenance activities, in-service information about the functional or operational performance of the product through life is often useful in providing validation and feedback of knowledge assumed at the design stage. Experience in service allows the assumptions made in the design process to be refined and corrected, and this new information in turn informs the next design process. However, knowledge and information gained from in-service experience is found to be consistently under-utilised in decision-making in design and development (Fundin, 2003). Increasing pressure for design right first time due to outsourcing and global competition means that there are greater incentives for companies to learn about their products in service. Increasingly common practice in aerospace industry, non-trivial experiences may also be recorded in best practice and lessons learned databases for future reference (Lowe et al., 2003, NASA, 2007). The main aim of 
lessons learned systems is to support sharing and distribution of knowledge (in the form of lessons learned) to the wider organisation in order to avoid repeating the same mistakes. They are usually generic and are not just limited to supporting in-service feedback but also other activities within the organisation.

A common means of codifying maintenance and service events is through the use of semi-structured documents, usually generated from standard forms or based on templates. These records may be generated from manual or digital form entries that are subsequently stored. Each instance of the records is usually referenced using unique identification systems such as serial numbering. Information contained in these records generally is a combination of mandatory fields such as service engineer's name, date, equipment attended, customer's name, site and free-text describing the problem and solution. Alternatively, electronic and online reporting systems such as Computerised Maintenance Management System (CMMS) or proprietary e-maintenance systems may be used with associated workflow management and events logging capabilities. For example, when a customer reports a problem through a phone call to a service centre, this issue is logged automatically and becomes a live issue that is managed electronically. These systems allow companies to manage and systematically respond to issues thus potentially improving customer relationships. Some systems also allow automatic user information capture, correspondence, reminders and archiving. The information within these systems may be structured ${ }^{1}$ (with predefined fields such as date, issue, customer, location etc.), but it is fundamentally different to the numerical data provided by sensors.

As semi-structured records the terminology and content of the maintenance and in-service information is subject to significant variance. There are opportunities for improving the information organisation aspect related to the service and maintenance records in the electronic form by abstracting this information into broader, aggregated and consistent viewpoints. In the immediate term, the abstracted viewpoint serves as a browsable organisational scheme for the retrieval of similar past issues and their resolution, thus reducing response time to urgent in-service issues. This gives a significant advantage to the in-service engineers as demands for decisions to be reached in much less time, such as stipulated in modern aircraft contracts. In the longer term, the organisation scheme may allow for repetitive systemic issues to be highlighted using statistical analysis and data mining to reveal emergent patterns or correlations in the collective data set that may not have been apparent previously. Such indications may help engineers to prioritise root cause finding and development of solutions to rectify them. The paper describes a faceted classification approach for organising semistructured records to enhance retrieval and also to facilitate knowledge discovery from the records. The approach can be described as a structured text mining involving records preparation, construction of the classification schemes and data mining from the records using OLAP-like approach. The method is demonstrated with an application in an aerospace case study.

\section{Literature Review}

Two distinctive strategies are typically adopted in searching and subsequent retrieval of information. The first approach involves term search techniques with the user entering a query or keywords that initiates a search for documents in the collection which are likely to be relevant to the user. Methods for matching the query to the document content (or the document index) are then carried out at search time. A variety of methods can be used to indicate relevance of document to query including Boolean, vector and probabilistic models (Baeza-Yates and Ribeiro-Neto, 1999). The biggest weakness in term search approach is that it relies greatly on the user formulating the suitable query to the targeted document corpus. Technique such as search expansion using thesauri has been used to improve querybased retrieval (Tikk et al., 2002). The second approach involves browsing documents that are organised, a priori, according to some predefined structure or classification schemes. The user browses the categories when searching for information. This approach requires more effort up-front in deciding and creating meaningful classification schemes, which may be difficult sometimes due to

\footnotetext{
1 'Structured' data or information is that which is represented electronically in a way that makes it interpretable to a computer, such that a computer can understand what to do with it when it is encountered.
} 
viewpoint dependency (which is addressed to some extent in faceted classification as discussed later). Ontologies have also been applied for improving information organisation and retrieval in various domains. An ontology, which is a specification of a conceptualisation, defines all the entities (objects or concepts) that are of interest in a domain and the relationships that connect these entities together, usually in some formal and preferably machine-readable manner (Hendler, 2001). Both classification and ontological approaches rely on the identification and abstraction of key concepts and their relationships from the underlying document corpus, thus requiring more efforts in construction and maintenance. However, they provide added advantages over the term search strategy in information retrieval as the abstracted schemes provide a browsable structure to facilitate retrieval as well as the analysis to deduce common patterns across the assigned concepts (or categorisations). This method is particularly useful when a user is unfamiliar with the domain terminology.

\subsection{Information Classification}

Classification is achieved by arranging objects into classes which can further be divided into subclasses according to some kind of principles of division. A class or subclass is a group of objects which share a particular set of attributes, with no other objects having this particular set of attributes. The purpose of classification may be analytical, i.e. to provide a systematic understanding of physical phenomena such as the biological classification for species of organisms. The famous Linnaean taxonomy uses five hierarchical ranks: class, order, genus, species, and variety. Another purpose of classification is for management of documents or information. The origins of modern approaches towards document classification are founded on principles originally developed by library scientists (e.g. Foskett, 1996, Rowley and Farrow, 2000, Taylor, 1992).

Information classification involves the development and use of schemes for the systematic organisation of knowledge (represented as information) to facilitate retrieval. There are three general types of classification schemes (Taylor, 1992):

- Enumerative - a scheme based on the concept of a universe of knowledge that is divided into successively narrower and more specific subjects.

- Synthetic - is a scheme in which new classes can be developed for new topics that are not already listed and establishes logical rules for dividing topics into classes, divisions and subdivisions.

- Analytico-synthetic (or faceted) - assigns terms to individual concepts and provides rules for the local cataloguer to use in constructing classification headings for composite subjects.

Enumerative classification seeks to list exhaustively all possible subclasses of interest in a particular class. The Library of Congress Classification (LCC) and the Universal Decimal Classification (UDC) are primarily enumerative. The major weakness with the enumerative schemes is that it imposes some subjectivity to the principle of division used, and introduces issues with viewpoint dependency. The number of specific subjects that have to be related in a classification (i.e. a field of knowledge) can potentially be infinite. For instance, a compound subject of "statistics for scientists and engineers" can be ambiguously classified under subject "statistics" and/or target audience "scientists" or "engineers". A further weakness of the enumerative scheme is the need for regular revision to deal with new and emerging subject that has not been considered during the construction of the scheme. This method requires significant maintenance efforts if the subject of classification is dynamic and changes regularly. These weaknesses are, to varying degrees, addressed by faceted classification approaches.

Although not the inventor of facet analysis, Ranganathan is credited as the first to systematise and formalise the theory (Foskett, 1996, Rowley and Farrow, 2000, Vickery, 1975). According to (Taylor, 1992), facets are clearly defined, mutually exclusive, and collectively exhaustive aspects, properties or characteristics of a class or specific subject. Facet analysis, applied to the presentation and physical layout of thesauri, involves (Rowley and Farrow, 2000):

- Identifying sets of terms representing concepts. This involves the identification of multiple 'simple' concepts from the compound subjects (s) that describe a document.

- The grouping of the terms representing the simple concepts into a number of mutually exclusive categories (called facets). 
- Organising the facets into a limited number of fundamental categories - these fundamental categories can be viewed a being different types of classification schemes. Thus the process of organising facets is essentially analogous to a process of classification scheme construction.

When a new subject is needed, a classifier using an enumerative scheme will have to wait until the scheme provides a term for that particular subject. In the case of a faceted scheme it is much easier to combine already-existing terms to form a new subject. In addition, because the concepts or facets for each classification scheme are compiled independently of each other, this ensures that the terms chosen can be developed and maintained independently. A key strength of the faceted classification is that it allows for multiple viewpoints to be represented through concurrent schemes.

\subsection{Data and Text Mining}

Data mining has been used for extracting new and potentially interesting patterns from highly structured data. Methods in machine learning, pattern recognition, and statistics are used extensively in data mining to discover features of interest from databases. Two types of analysis are generally considered in data mining application. The first approach creates models or predictive patterns for making an educated guess about the value of an unknown attribute given the values of known attributes (Witten and Frank, 2000). This modelling method is also known as the supervised learning, using techniques and algorithms like classification (decision tree) and numerical prediction (regression). On the other hand, the second approach aims to create models that do not solve a specific problem but simply to provide some description of the domain of interest. This modelling method is also known as the unsupervised learning, where techniques and algorithms such as clustering and association are employed in the exploratory exercise. Decision tree seeks to generate a series of logical rules that prescribe the performance of a series of instances or cases given their characteristics. The algorithm divides the set of instances into separate segments, each of similar performance, based upon the values of certain characteristics as seen within the full range of instances. By dividing these segments into smaller and smaller partitions, ultimately to the point where each member of a group has identical performance, a number of logical rules are generated which relate the value of the characteristics to performance. Association rule induction can be useful to describe interesting relationships between variables (or dimensions) from the data. The boundary between the two types of analysis is, however, not sharp as depending on the degree of the model interpretability some of the predictive models can be descriptive, and vice versa (Fayyad et al., 2006).

Text mining is about looking for patterns in natural language text, and may be defined as the process of analysing text to extract information from it for particular purposes. Related research in Natural Language Processing (NLP) seeks to use computers to automatically understand human languages. To date some progress has been made in this area, particularly within the subjects of bioinformatics where samples of human language are converted into more formal representations that are easier for computer programs to manipulate. As oppose to NLP, text mining focuses on extracting a small amount of information from text algorithmically and discovering relationships from the extracted information in a similar manner to data mining. The wider data mining process also includes data preparation, data selection, data cleaning, incorporation of appropriate prior knowledge, and proper interpretation of the results of mining, to ensure that useful knowledge is derived from the data (Fayyad et al., 2006). Similarly, text mining process generally consists of document collection, text preparation and mining operations which may include feature extraction, classification and clustering. These activities are particularly useful, for example, for the identification of key phrases in the absence of any thesaurus or controlled vocabulary in unstructured text (Witten and Frank, 2000). The overall process of turning unstructured textual data into high level information and knowledge is sometimes known as knowledge discovery in text. 


\section{Faceted Classification Using Waypoint}

Faceted schemes have two notable weaknesses, both related to the manner in which a user may interact with a constructed faceted classification. In cases where a set of documents are retrieved via concurrent selections from multiple facets, it is difficult to rank the documents in order of relevance as it is unclear how to assign suitable relevance across these different facets - it is possible that a given document will have great relevance to one selection, and a less strong (but still significant) relevance to a separate selection. It is also possible to make concurrent selections across different facets which would be infeasible in practice, where the set of returned documents under a selection for one facet might not intersect with the set of returned documents from a concurrent selection under a different facet. This second weakness, and to a lesser extent the first weakness, may be addressed using a system entitled Adaptive Concept Matching (McMahon et al., 2002) which has been implemented within the Waypoint faceted classification environment.

\subsection{Adaptive Concept Matching ${ }^{\text {TM }}$ (Adiuri Systems)}

Adaptive Concept Matching (ACM) describes a method in which a series of concurrent selections from different facets may be built up incrementally to avoid infeasible combinations of concepts. A faceted classification scheme is populated by a document corpus, and users are invited to make a series of selections from each facet. As the first selection is made, all documents that are not relevant to that selection are removed from the returned document set. This process is repeated, where the returned document set is gradually pruned of non-relevant documents. It is perhaps conceptually simpler to think of each selection as being a query in its own right, and that a complex query can be incrementally constructed via a graphical interface (McMahon et al., 2002). The benefit of the graphical interface is that the size of returned document set given at each incremental step can be evaluated and displayed, thus providing the user with an impression of the discrimination of their search. This display is extended to include the number of surviving documents contained under each class, making it possible to remove all unpopulated classes from the visual interface, preventing the user from making selections that would return no documents thus preventing infeasible queries.

As opposed to Document Management systems, the Waypoint environment does not collect and store documents, instead it is an environment within which a user may explore document corpora and retrieve relevant documents from their original source and in their native format. As the system operates separately from the document management, and thus has no mechanism through which to enforce manual classification at the point of document creation or archive, it is essential that documents may be automatically classified within the faceted scheme. Such an approach also extends the applicability of the system to legacy documents. The automated classification is conducted via the use of constraints, syntactic rules that identify key terms within a document which indicate its relevance to a category within the classification scheme (Figure 1). A hypothetical example of such a constraint is given below.

IF document contains Term 'Landing Gear' AND Term 'Bush' OR Term 'product SN XXXXX' THEN document is relevant to concept 'Landing Gear Bush'

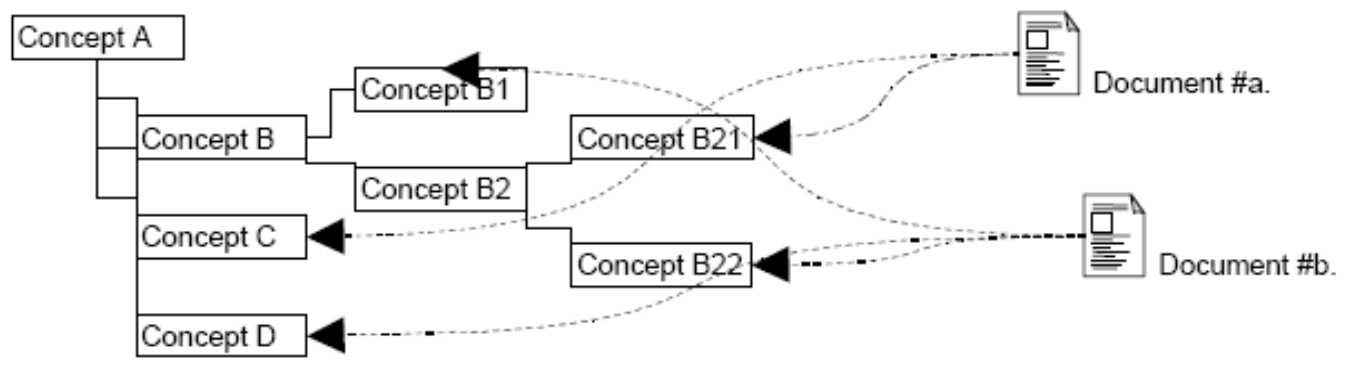

Figure 1 Faceted classification associating documents to categories under each facet (Waypoint, 2006) 
These constraints may be constructed for each concept node within the concept map, and is typically carried out concurrently with the construction of the faceted scheme - the scheme is itself a distillation of terms that appear in the documents (as concepts). The association of a document to a concept node through constraints is explained in the following section. This construction does not have to be conducted upon all documents, a subset of documents may be interrogated for this purpose (called the training set). It is also possible to automatically parse the documents to extract all significant terms (for example, by comparing the prevalence of certain terms within a document as compared to the prevalence within the overall corpus, referred to as "term frequency - inverse document frequency" (Baeza-Yates and Ribeiro-Neto, 1999)) and aggregate those key terms to form concepts. Methods for assisting concept identification will be discussed for the case study in later section although controlled vocabulary can greatly assist in this task.

\subsection{Exploiting Document Structure}

'Structured' data or information is that which is represented electronically in a way that makes it interpretable to a computer, such that a computer can understand what to do with it when it is encountered. Structured data can be found in such things as relational databases and in files which are tab or location delineated. Here the data are organised into a data structure according to the relationships and data type definitions prescribed by a data model. Unfortunately, much of the information generated in the course of the engineering process (in such things as reports, communications, procedures, catalogues, etc) is not only unstructured - making it difficult for electronic information systems to handle - but by its nature difficult to structure in its entirety. Nonetheless, it is possible to bring some order and machine-interpretability to unstructured information by making it semi-structured. This is done conventionally using such things as headings, paragraphs and sections (physical structure) and more recently by tagging or marking up interesting elements of the content explicitly using purpose-built formal languages such as HTML or XML (WC3). For instance, it may be possible to identify commonly occurring information elements, and develop a scheme or model by which these information elements in a document can be partially structured (and indeed, new documents constructed).

Particularly useful are the semantic (that is, meaning-bearing) information-bearing elements of content. One document may have many semantic dimensions, each of which is represented by a different structure. Revealing the semantic content of information allows information to be searched for based not on conventional pattern-matching techniques, but on meaning of the content. The process of making semantic structure explicit by marking up information content effectively means that documents can be decomposed into smaller and meaningful chunks. Waypoint specifically makes use of the explicit structure of XML document when making an association between a document and a concept. This is done by specifying the part of structure to which the constraints apply. Figure 2 illustrates the following rule in associating a structured document to a concept node in the concept map with the following rule.

Document is relevant to concept 'Damage' IF AND ONLY IF document contains Term 'Damage' within the 'Description' field 


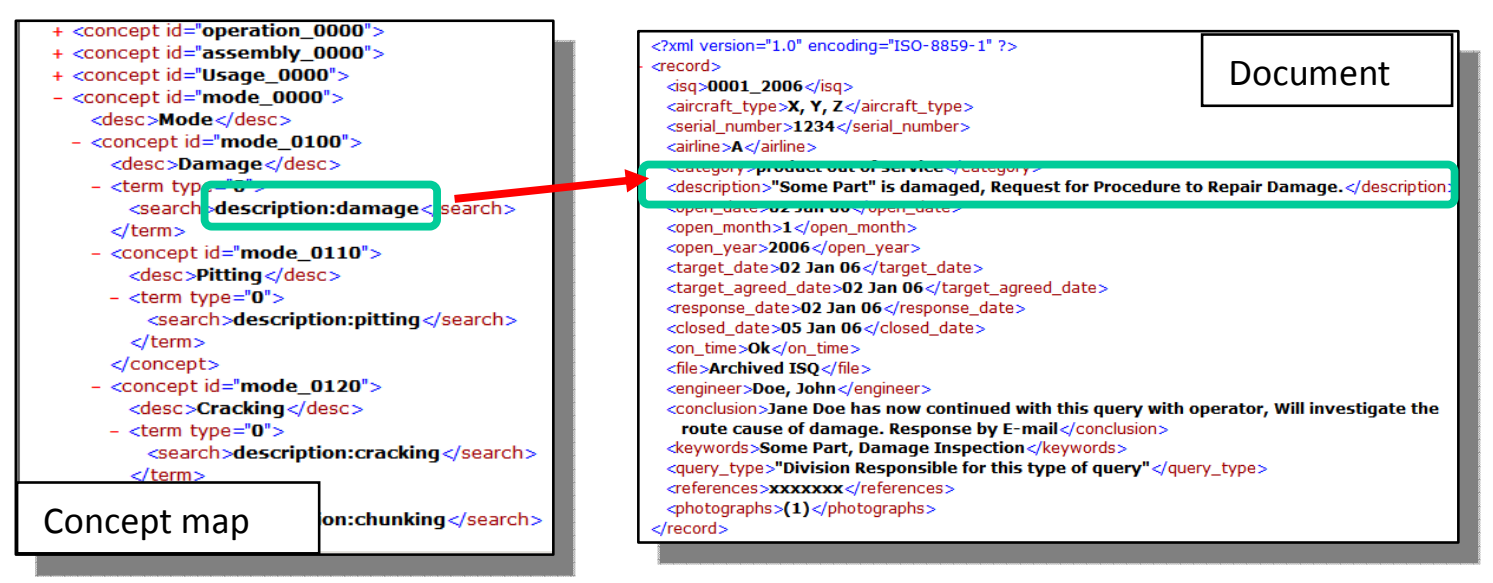

Figure 2 Association of a structured document to a concept node in the concept map

\section{Aerospace In-Service Records}

In face of ever reducing time to respond to unplanned in-service events, the In-Service Support (ISS) teams rely greatly on past experiences to expedite their responses to the aircraft operators. Previously, they depend greatly on knowledge of the work context and company's organisation structures, along with a keyword search on the text descriptions to retrieve and reuse similar past cases. An example scenario may be searching for In-Service Queries (ISQ) based on the engineer responsible or the dates from memory of a similar event. Although the approach may be efficient to the familiar users, it is problematic to users outside of the ISS teams (such as the designers). By classifying the ISQ records according to additional facets such as assembly/component, failure mode, operational phase etc. retrieval by users who are unfamiliar with the context can be facilitated. For instance, the databases become more useful for the designers to interrogate for component-related failure modes, operationalinduced issues etc. This added advantage was also recognised by the ISS engineers.

\subsection{Construction of the Classification Schemes}

In practice, the creation of classification schemes or schedules involves a great deal of intellectual effort. Two approaches, the 'top-down' or the 'bottom-up' approach may be used. The 'top-down' approach is usually simpler where the scope of the domain is considered, and these are divided and further sub-divided according to a consideration of specific distinguishing characteristics. Such an approach presupposes that the classifier's view of the world is both complete and accurate and that they can clearly identify which characteristics of a document to use as points of division. The more complex approach is referred to as a 'bottom-up' approach, which may be considered as an analyticosynthetic approach. This does not require an accurate or complete view of the domain in question, rather it depends upon adequate possession of the documents available within that domain. The view of the domain is obtained by distilling the essential characteristics or concepts of each document, which define the scope of the domain, and defining the classification scheme according to a natural grouping of these distilled concepts. Faceted schemes may be constructed via the use of both approaches, however the bottom-up approach ensures independence between concepts across facets, that there is no duplication within the scheme, and each given document is described by a combination of concepts that are adequate to completely describe and identify that document.

The method of identifying key concepts, terms and relationships within documentation and structuring these into a classification scheme can also be influenced to some extent by warrant (Beghtol, 1986). Many types of warrant have been suggested, including user warrant, scientific warrant, educational warrant and cultural warrant. For example, domain experts could create classification schemes based upon the scientific warrant whereas the user warrant aims at supporting the end user. The literary warrant describes the practice of constructing a classification scheme based upon the specific content of literature (Hulme, 1911). In terms of applicability, analytico-synthetic schemes rely upon literary warrant where the concepts which are contained within the document corpus are identified beforehand 
and the scheme arranged to fit these concepts. In this case study, the faceted schemes were constructed bottom-up based on literary warrant, which was then compared, refined and verified by domain experts.

From the ISQ database with many thousands of instances, the records for a selected number of years were scripted into structured XML documents with each piece of information tagged (shown in Figure 2 "Document"). An XML document can be viewed as the meta-information about an ISQ instance, where hyperlinks to the original documents such as reports, photographs and e-mails may be embedded for future retrieval. As mentioned previously, the structure improves the effectiveness in the Waypoint classification, such that rules can be coded under each tag/field. Some of these tags were inherited from the current ISQ database, particularly those referring to the airline, aircraft type and the ATA chapters which naturally become facets by which to organise the documents. These facets however, mainly relate contextually to the ISQ instance (except the ATA chapters which reflect the subsystem and functions to a limited extent). They do not sufficiently describe the technical detail of the issues. Some fields may contain numerical information such as the dates, the flight cycles and hours, which can be classified according to ranges specified in the rules. Nevertheless, information being described in the brief descriptions (free text and usually in an incomplete sentence) was found to be useful in deriving additional facets by which to classify the documents. The descriptions provide content-based information to the issue such as the failure mode, assembly, operational phase, flight type, topological location on the aircraft ${ }^{2}(\mathrm{RH} / \mathrm{LH}$, rib \#, leading/trailing edge) etc. Table 1 summarises the concepts and facets that are distilled from the description in addition to the inherited ones.

Table 1 Concepts and facets distilled from the "description" of Fuel Systems and Landing Gear ISQ records in addition to the inherited facets

\begin{tabular}{|c|c|c|}
\hline Facets & Categories & Concepts (examples of) \\
\hline \multirow[t]{7}{*}{ Assembly: } & Landing gear & $\begin{array}{l}\text { shock absorber, downlock actuator, uplock roller, grounding stud hole, sliding } \\
\text { piston, sliding tube, lower bearing, lower harness, tyres, axle, seal, aft pintle } \\
\text { pin, bogie harness wire, retraction actuator, wheel, bushes, disconnect boxes, } \\
\text { freefall system, bell crank bush, primary seal, steering feedback sensor, } \\
\text { chrome plate, wing bracket }\end{array}$ \\
\hline & Fuel system & $\begin{array}{l}\text { transfer valves, dry bay drain hole, trim tank harness, centre tank pump, inner } \\
\text { tank pump, refuel panel plug, outer LHS tank, outer RHS tank, pipes, vapour } \\
\text { seal, probes, engine feed pipe, standby pump, collector cell, water scavenging } \\
\text { system }\end{array}$ \\
\hline & Steering system & turnbuckle \\
\hline & '"'software & FCMC, BSCU \\
\hline & $\begin{array}{l}\text { Air } \\
\text { conditioning } \\
\text { system }\end{array}$ & N/A \\
\hline & Engine & N/A \\
\hline & Brake system & N/A \\
\hline \multirow[t]{4}{*}{$\begin{array}{l}\text { Failure } \\
\text { mode: }\end{array}$} & Damage & $\begin{array}{l}\text { Corrosion } \\
\text { Mechanical: cracking, wear, pitting, dent, rupture, delamination, chafing } \\
\text { Heat: overheat, high temperature } \\
\text { Chemical } \\
\text { Other: manufacturing, leakage, spillage, debris contamination, water } \\
\text { contamination }\end{array}$ \\
\hline & System & fault, complete failure, partial failure \\
\hline & Event & collision, bird strike, lightning \\
\hline & General query & $\begin{array}{l}\text { agreement to defer, agreement to modify, update procedure, clarification, } \\
\text { resolution }\end{array}$ \\
\hline Operational & In-flight & cruise, take-off, landing \\
\hline
\end{tabular}

\footnotetext{
${ }^{2}$ This information is more pertinent in the Wing Structures ISQ.
} 


\begin{tabular}{|c|c|c|}
\hline phase: & & \\
\hline & $\begin{array}{l}\text { Ground } \\
\text { operation }\end{array}$ & '"' refuelling '"' \\
\hline & Maintenance & $\begin{array}{l}\text { jacked, cleaning, overhaul, lubrication, fuel leak, test, inspection, brake } \\
\text { bleeding, installation }\end{array}$ \\
\hline Flight type: & Revenue flight & \\
\hline & Test flight & \\
\hline & Ferry & \\
\hline
\end{tabular}

Central to the effectiveness of the information organisation system depend on the information quality and the classification schemes used. By performing the bottom-up approach from a subset of the document corpus a number of key concepts were found that are consistently used by the ISS engineers, which provides the domain vocabulary for evolving taxonomy. Since the quality of legacy records and the classification schemes may not be adequate in the first attempt, continuous evolution and refinement of the classification schemes and the reporting of newly identified key information is necessary to improve utility of the system. For instance, some common concepts may not be readily identifiable from the records due to variation in the way of reporting. Some of the issues include:

- Inconsistency in referring to the product, failure mode, operational environment etc. in the description

- Information may be reported at different level of granularity e.g. damage/cracking

- Ambiguous terms and synonyms e.g. damage/broken, cracking/chunking

- Acronyms and abbreviation e.g. MLG/Main Landing Gear; assembly/assy

- Spelling variation and errors, e.g. centre/center

- Missing or implied information in the description field

○ "MLG Pin Assembly" - the issue affecting the product is not explicitly reported

○ "Lower Bearing Corrosion" - the main assembly of the part is not reported.

From the information contained in the Fuel Systems and Landing Gear database for the selected number of years, the full classification show sparse records in some of the facets, inevitably for those derived from the description, as summarised in Table 2. The flight type is the least frequently quoted information in the records with only $1.03 \%$. The operational phase and the failure mode have 30.14 $\%$ and $25.79 \%$ respectively of the whole document set successfully classified. However, $58.79 \%$ of the records contain information about the product assembly and components that is being queried. The statistics provide an indication of the inconsistency in the reporting of queries that may be important to learning about the causes of in-service issues.

\section{Table 2 Statistics of the Classification}

\begin{tabular}{|l|l|r|}
\hline Facets & Inherit/From Description & \% of Classified Documents \\
\hline ISQ Date & Inherit & 99.75 \\
\hline Category & Inherit & 99.07 \\
\hline ATA Chapter & Inherit & 90.27 \\
\hline Aircraft Type & Inherit & 90.00 \\
\hline Assembly & From description & 58.79 \\
\hline Query Type & Inherit & 44.88 \\
\hline Operational Phase & From description & 30.14 \\
\hline Failure Mode & From description & 25.79 \\
\hline Flight Type & From description & 1.03 \\
\hline
\end{tabular}

In order to facilitate the construction of classification schemes from text description, methods in text mining were considered in order to extract key phrases that appear frequently in the text to represent concepts (and the corresponding rules/constraints). As previously mentioned, the information in the description field is critically important to the engineers as it provides a summary to help retrieve more 
complete information from the original correspondences and technical reports that are linked to the ISQ instances. These documents are often in scanned PDF format, albeit for legislative reasons, are not readily treatable.

\subsection{Text Mining Methods}

As the classification schemes were to be constructed based on the literary warrant, i.e. evolved from the underlying content of corpus, text mining methods are suitable to facilitate the automated identification of key phrases from text. In information retrieval, methods such as stemming are often used to deal with common lemma of words. Additionally, spelling variation, abbreviation and acronyms can be dealt with using dictionary and domain knowledge to link between similar concepts (Wren et al., 2005). The Waypoint system includes such functionality by incorporating the OpenSource Lucene indexing and search engine library (Apache, 2006). This library has a number of different document analysers which cater for different forms of such treatment. For the Wing Structures database, facets related to the topological location of the fault were particularly important. Although the facet was inherited from the original company database, the records in these fields were only sparsely populated. Improving the records will improve the classification as the information is valuable for learning about the characteristics of the in-service issues. Information was extracted from the unstructured description field to populate the classification schemes using a dictionary of terms that are expected in each concept node. The records with missing information are matched against those terms and are filled-in. Pre-processing operations such as this can improve the accuracy of the association of the documents to the rules/constraints in the concept map.

As the domain is quite restricted, many concepts are repeated in the records. Although methods proposed in Natural Language Processing (NLP) such as Part-of-Speech (POS) tagging appear to be relevant in the first instance, they are found to be less useful in dealing with technical reporting which does not conform to standard grammatical rules (Ciravegna, 1995). As shown in Table 1, the concepts need to be grouped into classes to provide a logical classification structure (either enumerative or faceted schemes). For instance, aircraft types can be grouped according to their families of variants which share similar characteristics e.g. single/twin aisle, medium/long range, twin/four engine. By doing so, behaviour displayed by the families of aircrafts could be discriminated in the classification. As such, the means of aggregating the concepts into classes and the granularity of the classification have significant influence on ability to make inference from the patterns and trends in the records. Although methods like WordNet and Formal Concept Analysis have been used with some success in a number of areas (Stoica and Hearst, 2006, Cimiano et al., 2004), it was found that domain knowledge is still essential to provide meaningful abstractions at present.

In this case study, statistical methods were used to analyse the frequency of collocated words to extract most frequent word clusters. High frequency word clusters are a good indication of common phrases referring to some meaningful concepts. The concepts extracted from the ISQ records reflect the domain vocabulary used by the in-service engineers. Table 3 shows the results of word clusters with a minimum frequency of 10 . The clusters shown are generated from indices in concordance with the keyword "tank". The number of words in a cluster (known as window) and the minimum frequency were arbitrarily set depending on the characteristics of the corpus. The significance of the word clusters as concepts can be improved by breaking the sentence at punctuations and common stop words. Also as can be seen from the table, automatically extracted concepts can be hierarchically arranged into the main assembly, sub-assembly and component of tank with sufficient level of accuracy. This is similar to the concept pairs heuristics proposed by Yang (2004).

Table 3 2-5 word clusters in concordance with "Tank", minimum frequency $=10$

\begin{tabular}{|l|l|l|}
\hline Main assembly (freq) & Sub-assembly (freq) & Components (freq) \\
\hline Fuel centre tank (10) & Tentre tank (110) & Tank FQI (12) \\
& Center tank (41) & Tank fuel (44) \\
\hline Fuel trim tank (12) & Fuel tank (69) & Tank harness (24) \\
\hdashline LH inner tank (12) & Inner tank (111) & Tank indication (12) \\
\hdashline RH inner tank (10) & Surge tank (10) & Tank inlet (21) \\
\hdashline RH outer tank (10) & Trim tank (220)
\end{tabular}




\begin{tabular}{|l|l|l|}
\hline & & Tank inlet valve (19) \\
\hline & Outer tank (75) & Tank low (16) \\
\hline & Wing tank (55) & Tank NTO (12) \\
\hline & & Tank overflow (12) \\
\hline & $\begin{array}{l}\text { Tank pump (33) } \\
\text { Tank pumps (12) }\end{array}$ \\
\hline & Tank transfer (35) \\
\hline & Tank vent (10) \\
\hline
\end{tabular}

\subsection{Retrieval and Knowledge Discovery}

In the Waypoint implementation, the ISQ instances (XML documents) were classified according to the faceted schemes that were constructed as previously described. Through the interface, a number of concepts in the faceted schemes can be selected to form a compound query. For instance, if the user is interested in corrosion on aircraft type X, he/she will select the concept " $X$ " under the facet Aircraft Type and the concept "Corrosion" under the facet Failure Mode. The returned documents are pruned from the system leaving only relevant records that satisfy the conjunction of the selected concepts. At this point, a much smaller set but highly relevant documents can be retrieved as a list by clicking on the 'Results' button on the top right hand corner as illustrated in Figure 3(a). A list of results that are only relevant to both concepts will be displayed as shown in Figure 3(b). The display can be customised as well as hyperlinked to other sources of documents that are relevant to those ISQ instances. Due to confidentiality, the actual numbers of documents and some fields are not shown in the figures. The use of Dynamic HTML (DHTML) allows the output to be easily formatted or inserted into other applications or web pages. If the returned document set is still too large, the user can introduce further constraints (for example by selecting the concept "Main Landing Gear" under Assembly) to reduce the set further and increase precision. This way, the faceted classification schemes help to quickly identify and retrieve experiences and resolution to past issues based on a combination of the concepts selected.

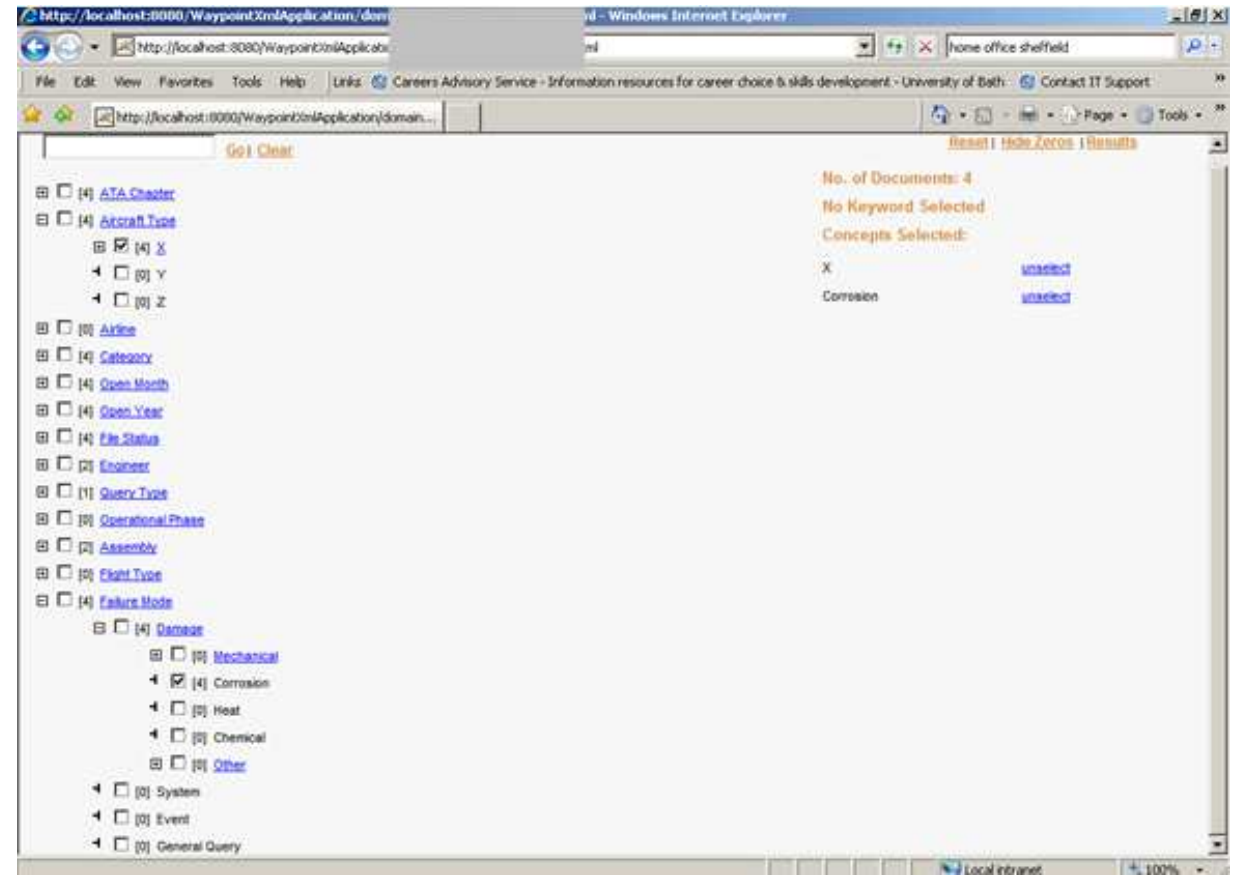

(a) 


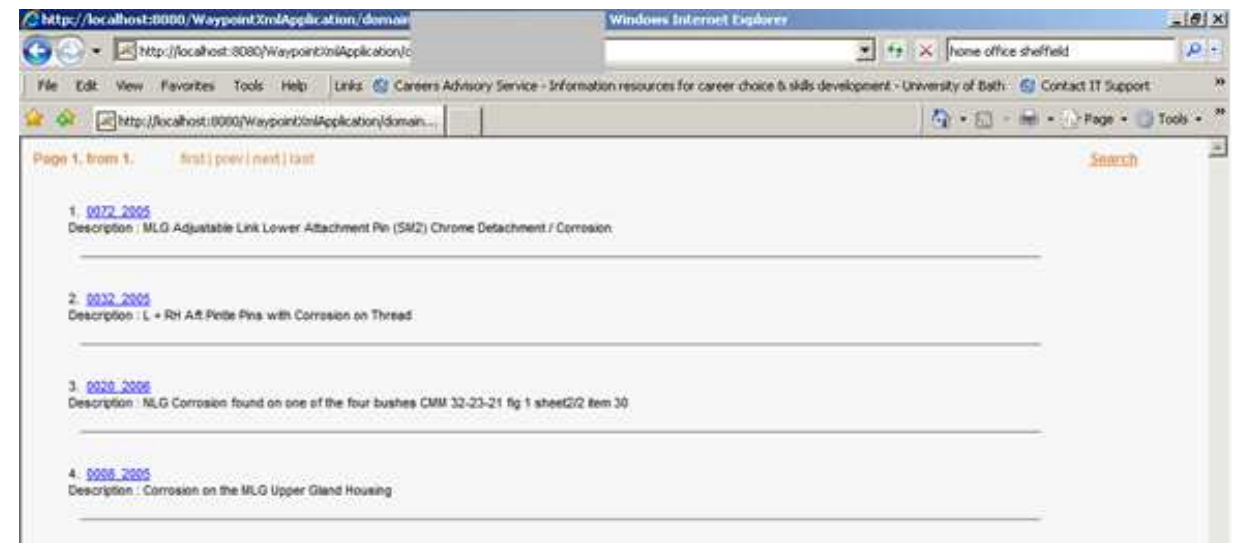

(b)

\section{Figure 3 (a) A Waypoint faceted classification interface (numbers do not reflect actual ISQ records) (b) List of documents relevant}

As the users interactively browse through the classification tree and introduce constraints (by selecting more concepts) in Waypoint, the document set is pruned and the remaining relevant document set is updated dynamically. The document count is reflected in the numbers next to each node (at all levels of the tree), where only those documents classified under that node and are also relevant to the current selected concepts are displayed (Figure 3 (a)). For example, if the user wants to find out about the failure modes that most frequently affect the main landing gear of aircraft $X$, with these two nodes selected, he/she can navigate to the Failure Mode facet. The ISQ documents will be distributed across the four classes (Damage [a], System [b], Event [c] and General Query [d] as shown in Figure 3(a)) with the number of relevant documents displayed next to each node in brackets. This allows the user to infer dominant class(es) of failure mode reported about the main landing gear of aircraft X, say Damage [a]. By expanding the Damage class, all the active (still relevant) concept nodes are displayed with numbers next to each one of them (Figure 3(a)). Then, the user might also want to limit the results to those reported in a particular year or month. At any stage, the concepts with no document count against them can be hidden or shown depending on the users' preference. The number of documents at a parent node may be equal to or greater than the sum of documents for all the children nodes as some instances may be classified under more than one concept nodes in the same facet. For example, an ISQ instance that reports both mechanical and heat issues will be classified and counted against both the nodes but only counted once for the Damage class (Figure 3(a)). This flexibility allows for documents to be associated to concept nodes at any level of the tree to cope with the varying level of abstraction in the reporting of ISQ.

By browsing through the faceted schemes in Waypoint, one might be able to discover classes of inservice issues that are systemic such as design-induced (if there is apparent correlation between a component/topological location and a failure mode), operation-induced (if there is apparent correlation between an operational phase/event and a failure mode) and use-induced (if there is apparent correlation between a flight type/route/aircraft operator and a failure mode) issues. Correlation can be indicated by higher than average number of instances between two or more concept nodes. Although the correlations do not necessarily indicate an underlying problem, by indicating that it is a frequent occurrence, it acts as a prompt for engineers to determine root causes, which may lead to understanding of the operating conditions practiced by different airlines (e.g. maintenance procedure, type of operation - short or long haul). Furthermore, it becomes possible to infer less apparent correlation such as between a time period and failure mode to suggest potential inherent reliability issues. The capability will help towards highlighting recurrent patterns and correlations between different facets across a large set of ISQ instances. Such effort could have been achieved in the past through manual tracking and compilation of data or through experience but it can be achieved interactively in the Waypoint environment. This mechanism can be used to provide evidence to the design team when formally requesting engineering change or in prioritising 
continuous development efforts as well as for analysing reliability of components and operational interruptions. Some of these insights may also be usefully reported as lessons learnt which might benefit future aircraft development programs. A unified knowledge management solution for learning from experience can potentially be realised given that the company has also implemented a software tool for capturing and sharing lessons learned.

\section{Discussions and Conclusions}

Information collected from service that are commonly of semi-structured form can be more effectively reused if subject to some organisation. The faceted classification approach described in this paper not only provides an improved retrieval mechanism to aid speedy resolution of ongoing inservice issues but also provides a potentially useful mechanism for learning from the collective instances. The faceted schemes allow the user to arrive quickly at a highly relevant set of results by selecting concepts relevant to the query. In addition, the facets derived from the content of the ISQ records are especially useful to a non-familiar user to browse for information. As a result, the system becomes meaningful to others to interrogate, such as designers looking for typical issues raised on a particular component of the aircraft. Perhaps more importantly, the faceted schemes also allow for patterns and trends in the records to be highlighted, either by browsing the classification tree or automatically detecting patterns using data mining algorithms. The patterns and correlations may suggest for systemic and repetitive issues observed in service, which can be used as objective evidence to prioritise root-cause finding and continuous development efforts. Although the role of learning and discovering knowledge still lies with the domain experts, this is in contrast to the present way of learning that depends on subjective and manual assimilation.

The construction of faceted classification schemes may involve significant intellectual efforts in defining the levels of granularity and the dimensions of information relating to the records that are subject to organisation. In doing so, faceted classification provides a mechanism for viewing information at different levels of granularity using the concept hierarchy similar to On-Line Analytical Processing (OLAP) (Hand et al., 2001). Waypoint in particular allows the user to interrogate for patterns and trends through its dynamic interface. Aggregations can be built by changing the granularity on specific dimensions (facet) and aggregating up data along these dimensions. For instance, the user can choose to look for patterns that are characteristics of a family of aircraft or of a specific aircraft model, of a sub-system or of a specific component etc. Further functionality of data mining can also be easily integrated with Waypoint to automate pattern identification from the semi-structured records. It can be anticipated that the construction of the hierarchical structure of the classification schemes will affect inferences that might be drawn because the number of instances in a class is determined by the lower level nodes that are associated with it. Therefore, selecting the suitable level of abstraction is not trivial and may require trade-off to be made between retrieval efficiency and usefulness for knowledge discovery.

To be most effective, the classification schemes and records have to be evolved and refined iteratively. With current records as the training set, a taxonomy that is based on the literary warrant can be constructed which will in turn guide the creation of high quality records through prescribed information entry. The use of a controlled vocabulary or taxonomy such as through prescribed data entry (e.g. through selection lists) would significantly enhance ability to classify, retrieve and mine the in-service information. The constraints and rules for the classification may also be refined to improve the association of the records to those abstracted concepts. Additionally, more value can be attained from in-service information if the records can be linked to manufacturing-related information, such as the batch number, supplier, etc. For example, using the part and serial number to allow for cross referencing the ISQ records to the Bill of Materials and manufacturing information can enhance prospects of knowledge discovery. Patterns related to the design and manufacture to the in use performance and events (such as the frequency of maintenance) can be investigated that may result in insights and learning. The previously unsuspected correlations can be made explicit so that proactive steps can be taken to incorporate learning and avoiding repeating similar mistakes in the future. This will also help designers to understand the performance limits of a design, by understanding how the 
product is being used. Designers in due course may want to adapt the design to changes in use that may be more costly to design but may be more economical to maintain in the extended lifecycle.

\section{Acknowledgement}

The authors gratefully acknowledge the funding provided by the Engineering and Physical Science Research Council (EPSRC) for the KIM Project (http://www-edc.eng.cam.ac.uk/kim/) under Grant No. EP/C534220/1 and the I $d \mathrm{MRC}$ under Grant No. GR/R67507/01 for the research reported in this paper. The contribution of Mr. Joe Cloonan and the In-Service Support team of Airbus UK is also gratefully acknowledged.

\section{References}

The Apache Software Foundation (2006) Apache Lucene -- Overview. http://lucene.apache.org/java/docs/index.pdf

Baeza-Yates, R. \& Ribeiro-Neto, B. (1999) Modern Information Retrieval, Harlow, UK, ACM Press Books.

Beghtol, C. (1986) Semantic validity: Concepts of warrant in bibliographic classification systems. Library Resources \& Technical Services, 30, 109-125.

Cimiano, P., Hotho, A. \& Staab, S. (2004) Clustering ontologies from text. Proceedings of the 4th International Conference on Language Resources and Evaluation. Lisbon, Portugal.

Ciravegna, F. (1995) Understanding messages in a diagnostic domain. Information Processing and Management, 31, 687-701.

Fayyad, U., Piatetsky-Shapiro, G. \& Smyth, P. (2006) From Data Mining to Knowledge Discovery in Databases. American Association for Artificial Intelligence, 17, FALL 1996, 37-54.

Foskett, A. C. (1996) The subject approach to information, London, Library Association Publishing.

Fundin, A. P., Bergman, B. L. S. (2003) Exploring the customer feedback process. Measuring Business Excellence, 7, 56-65.

Hand, D., Mannila, H. \& Smyth, P. (2001) Principles of Data Mining, Cambridge, USA, MIT Press.

Hendler, J. (2001) Agents and the Semantic Web. IEEE Intelligent Systems, 16, 30-37.

Hulme, E. W. (1911) Principles of Book Classification. Library Association Record, 13, 354-358.

Jardine, A. K. S., Lin, D. \& Banjevic, D. (2006) A review on machinery diagnostics and prognostics implementing condition-based maintenance. Mechanical Systems and Signal Processing, 20, $1483-1510$.

Lowe, A., McMahon, C. A., Culley, S. J., Coleman, P. \& Dotter, M. (2003) A Novel Approach towards Design Information Management within Airbus. ICED'03. Stockholm, Sweden.

McMahon, C., Crossland, R., Lowe, A., Shah, T., Williams, J. S. \& Culley, S. (2002) No zero match browsing of hierarchically categorized information entities. Artificial Intelligence for Engineering Design, Analysis and Manufacturing, 16.

NASA (2007) The NASA Engineering Network. Lessons Learned Information Systems (LLIS).

Ong, M., Ren, X., Allan, G., Kadirkamanathan, V., Thompson, H. A., Fleming, P. J. (2004) Decision Support System on The Grid. Int'l Conference on Knowledge-Based Intelligent Information \& Engineering Systems. New Zealand.

Rowley, J. \& Farrow, J. (2000) Organising Knowledge, Aldershot, Hants, UK, Gower.

Stoica, E. \& Hearst, M. (2006) Demonstration: Using WordNet to Build Hierarchical Facet Categories. ACM SIGIR Workshop on Faceted Search.

Taylor, A. (1992) Introduction to Cataloguing and Classification, Westport, CT, Libraries Unlimited.

Tikk, D., Yang, J. D., Baranyi, P. \& Szakal, A. (2002) Fuzzy relational thesauri in information retrieval: automatic knowledge base expansion by means of classiffied textual data. 6 th International Conference on Intelligent Engineering Systems. Opatija Croatia.

Vickery, B. C. (1975) Classification and Indexing in Science, London, Butterworth.

Waypoint (2006) Faceted Classification and Adaptive Concept Matching. Gemstone Business Intelligence Ltd.

Witten, I. H. \& Frank, E. (2000) Data Mining: Practical machine learning tools with Java implementations, Morgan Kaufmann. 
Wren, J. D., Chang, J. T., Pustejovsky, J., Adar, E., Garner, H. R. \& Altman, R. B. (2005) Biomedical term mapping databases. Nucl. Acids Res., 33, D289-293.

Yang, K., Jacob, E., Loehrlein, A., Lee, S., Yu, N. (2004) Organizing the Web: Semi-automatic construction of a faceted scheme. IADIS International Conference WWW/Internet. Madrid, Spain. 